\title{
Data Analysis for Emotion Classification Based on Bio-Information in Self-Driving Vehicles
}

\author{
Tae-Yeun Kim, ${ }^{1}$ Hoon Ko $\mathbb{D}^{,},{ }^{2}$ and Sung-Hwan Kim $\mathbb{D}^{1}$ \\ ${ }^{1} S W$ Convergence Education Institute, Chosun University, Gwangju, Republic of Korea \\ ${ }^{2}$ IT Research Institute, Chosun University, Gwangju, Republic of Korea \\ Correspondence should be addressed to Sung-Hwan Kim; shkimtop@chosun.ac.kr
}

Received 13 May 2019; Accepted 28 October 2019; Published 16 January 2020

Guest Editor: Hyunhee Park

Copyright (C) 2020 Tae-Yeun Kim et al. This is an open access article distributed under the Creative Commons Attribution License, which permits unrestricted use, distribution, and reproduction in any medium, provided the original work is properly cited.

\begin{abstract}
All persons in self-driving vehicle would like to receive each service. To do it, the system has to know the person's state from emotion or stress, and to know the person's state, it has to catch by analyzing the person's bio-information. In this paper, we propose a system for inferring emotion using EEG, pulse, blood pressure (systolic and diastolic blood pressure) of user, and recommending color and music according to emotional state of user for a user service in self-driving vehicle. The proposed system is designed to classify the four emotional information (stability, relaxation, tension, and excitement) by using EEG data to infer and classify emotional state according to user's stress. SVM algorithm was used to classify bio information according to stress index using brain wave data of the fuzzy control system, pulse, and blood pressure data. When $80 \%$ of data were learned according to the ratio of training data by using the SVM algorithm to classify the EEG, blood pressure, and pulse rate databased on the biometric emotion information, the highest performance of $86.1 \%$ was shown. The bio-information classification system based on the stress index proposed in this paper will help to study the interaction between human and computer (HCI) in the 4th Industrial Revolution by classifying emotional color and emotional sound according to the emotion of the user it is expected.
\end{abstract}

\section{Introduction}

In recent years, a new future technology called emotional artificial intelligence (AI) has emerged owing to the advancement of the fourth industrial revolution era. Particularly, AI-based emotional computing technology, which can interpret and analyze human emotions, is advancing rapidly owing to a convergence of information and communication technology (ICT) and cognitive science areas [1,2]. Accordingly, the human-computer interface (HCI) technology is becoming increasingly important, and along with the progress made in the studies on HCI, studies are being increasingly conducted on computer reactions based on emotion inference or user intention rather than computer reactions induced by direct inputs of the user [3]. Particularly, the brain-computer interface (BCI) technology analyzes, commands, or controls electroencephalography (EEG) signals in frequency domain, which were measured at a human scalp. Various studies are conducted to provide interaction between humans and computers by converging the user's emotion information and environment information based on the BCI technology $[4,5]$. Emotion is a cognitive ability of humans and a reaction for external sensory stimulation. Humans react emotionally according to various social and cultural factors and feel various emotions accordingly. Emotion recognition technology is a means of making intelligent decisions that can enable appropriate behavior by extracting information such as facial expressions or body gestures of a user based on emotion data [6]. As such, it is extremely important for computers to have emotion recognition capability to process human emotions through learning and adaptation in order to process the interaction between humans and computers more efficiently. In modern society, mental stresses such as various work-related stresses, conflicts in interpersonal relationships, and financial problems have emerged as social problems, and efforts are actively being made to improve the quality of physical and psychological life [7]. As such, it is important to measure the emotional stress level of users by using EEG and biosignals in objective numerical values in terms of psychological state, and efforts to relieve such stresses by recognizing the subsequent 
physical changes are necessary. As colors and music are formed in a short time and last for a long time in memory, they can play vital roles in understanding and analyzing human emotions. Therefore, this study aims to design a system that infers emotion by using biometrics of user, such as EEG, pulse rate, and blood pressure (systolic and diastolic blood pressure), and that recommends colors and music based on the emotional state of the user, i.e., the stress index. The system proposed in this paper recognizes the emotion of the user by learning and patterning the reactions appearing according to the user's emotional state and classifies the biometric emotional information according to the stress index. A fuzzy system was designed by using EEG data to classify the biometrics into biometric emotion information according to the stress index. Furthermore, it was designed to produce the pulse rate and blood pressure (systolic and diastolic blood pressure) data in single packets separately and to send them to the database. As such, after acquiring the biometrics, the biometric emotion information is classified according to the stress index through a support vector machine (SVM) algorithm. In general, various learning algorithms are applied to decision-support systems. However, as the biometric data used in this study consist of EEG, pulse rate, and blood pressure (systolic blood pressure and diastolic blood pressure) data, which have nonlinear data structures, the SVM algorithm was used to solve the nonlinear discrimination problem in a multilayer perceptron structure. Depending on the classified stress emotion, the data corresponding to the color and music values are classified. This paper is organized as follows. Section 2 discusses related research, and Section 3 describes the system configuration and design. Subsequently, Section 4 presents the performance evaluation and experimental results. Finally, Section 5 discusses the study's conclusions and directions for future research.

\section{Related Research}

2.1. Cognitive Science. Cognitive science examines the cognitive processes of humans and animals, such as perception, language, learning, and emotions. In this field, techniques are studied for applying these cognitive processes to the development of robots, electronic products, buildings, and so on $[8,9]$. Recently, as cognitive science has begun to attract attention, studies are being conducted on the detection, processing, and analyzing of various types of signals that are generated by bodily activities. In particular, studies on the structure and functions of the brain are being actively conducted. These studies utilize various biometrics for not only medical diagnoses, but also the examination of people's cognitive and emotional states through analysis via certain algorithms.

\subsection{Emotional Engineering. Emotions are high-level} psychological experiences that occur in people via their senses and cognition in response to an external physical stimuli. These are often-changing psychological effects that occur in people owing to complex feelings, such as comfort, pleasure, unease, and discomfort. These are the moods and feelings that are connected to expressive behavior during emotional reactions and physiological changes. Further, they are a dynamic aspect of thought. Emotional engineering is a field that studies emotions and deals with them in practical terms. This combines areas related to human psychological reactions and their applications, such as human factor engineering, cognitive science, behavioral science, pragmatic aesthetics, and environmental psychology [10]. Changes in people's psychological states are expressed externally in the form of feelings or emotions. In the past, people's psychological states and emotions were mostly analyzed and used as clinical data for medical diagnoses and treatments. However, they are currently used in a variety of fields, such as buildings that incorporate psychotherapeutic art and automatic lighting that reflects emotions, cars that prevent sleepiness, and robots that understand and respond to human feelings. Researchers are studying emotionally intelligent computer technology that can understand psychological states, feelings, and emotions. Emotionally intelligent computing allows computers to recognize people's feelings and emotions and to perform actions that are suitable to the circumstances. This entails autonomous systems that are able to perform suitable actions based on previous knowledge or the current psychological state. Recently, as wearable computing technology has evolved, it is able to measure biosignals such as electroencephalography (EEG), electrography (EMG), electrocardiogram (ECG), and galvanic skin response (GSR) more accurately, and produces improved recognition results regarding subjects' mental states, emotions, and physiological states [11].

\subsection{Biobased Emotional Cognitive Technology Trend. In} general, the emotions felt by people can be distinguished by the central nervous system or autonomic nervous system reactions, i.e., information such as ECG, EEG, skin temperature (SKT), and GSR. The biosignal emotion recognition technology, that has been studied so far, can be divided into studies using statistical methods and those using machine learning methods. Studies on rule-based techniques analyze many biosignals, and extract emotional features that express emotions. Then, threshold values that correspond to rules are set for each emotion, and emotions are classified according to these thresholds. Emotion recognition methods that use machine learning are based on techniques such as neural network (NN), support vector machine (SVM), k-nearest neighbor (kNN), multi-layer perceptron (MLP), Gaussian mixture model (GMM), decision tree (DT), and Baysian network (BN) [12-14]. For biosignal learning, it is necessary to collect a large amount of feature data and perform the training required for emotion recognition. Therefore, the amount of collected feature data and its reliability have a significant effect on the subsequent performance of the recognition system. However, most emotion recognition systems that have been studied so far use data with staged and exaggerated emotions. Because it is easy to artificially acquire learning data that includes emotions, this is a major factor that degrades the performance of emotion recognition systems in real situations. Therefore, the collection of reliable biometric data is considered necessary for biosignal-based emotion recognition. However, in biosignal collection, a user's emotional states can easily change according to the environmental conditions or the 
user's psychological state, and it is very difficult to perfectly recognize a user's emotions based on a single biosignal. Therefore, rather than emotion recognition using only one biosignal, researchers are investigating methods that use several biosignals in conjunction or use additional emotional measurement indicators, such as voice and facial expressions. Emotion recognition technology using brainwaves is one of the most actively studied fields in biotechnology. Unlike other biosignals, brainwaves show the state of the central nervous system. The alpha and beta waves that occur according to the state of brain activity are known to be related to emotion. Alpha waves generally indicate a stable or relaxed state, and these increase during positive emotions. On the other hand, beta waves increase during negative emotional states [14]. In addition to brainwaves, autonomic nervous system reactions occur when commands are received from the brain, and these indicate bodily changes when a person is surprised or encounters danger. These reactions include the heart beating rapidly, the facial muscles stiffening, the palms and back of the neck sweating, and changes in body temperature or the temperature at certain parts of the body. These bodily changes are controlled by the autonomic nervous system, and therefore emotional states can be inferred from biosignals by detecting autonomic nervous system reactions. Biosignals that can be measured following autonomic nervous system reactions include ECG, EMG, GSR, and SKT. Studies have used the heart rate variability (HRV) and heart rate (HR) to recognize emotions. A low heart rate indicates a relaxed state, and a high heart rate can indicate stress, frustration, and dissatisfaction. As such, the heart rate is often used for data analysis. Emotions can be classified by calculating the HRV from the heartbeat and extracting features. However, it is difficult to accurately recognize emotions from the heartbeat alone [15]. Emotion recognition via EMG has mainly consisted of studies on the recognition of emotions through the movement of facial muscles. The movement of facial muscles can be measured through an EMG of the face, and this can be used to measure high-stress tension and so on, and recognize emotions. In a tense/aroused state, the EMG increases, whereas in a relaxed state it decreases. However, the absolute levels of muscle tension vary according to the part of the muscle that is measured. As such, this signal requires precise measurement. GSR essentially refers to skin conductivity, which increases when sweat is present on the skin. This can be used as an indicator of stimulation or stress. The GSR amplitude increases during arousal or negative emotions. The reaction speed (latency) is quick during sensitive stimulation. The reaction sensitivity (slope) is large during sudden or sensitive stimulation. Therefore, GSR can be used as a good indicator for measuring negative emotions. SKT is different from body temperature, in that it is a temperature index for certain body parts. This is not a normal core biosignal indicator, but it can be used as a slow indicator of changes in emotional states. It is significantly affected by external environmental factors. In general, when the amplitude of the SKT signal is large, this indicates relaxed and pleasant positive emotions. When it is small, this indicates tense or uncomfortable negative emotions. Recently, there has been a sudden interest in many different fields in attempts to monitor image, voice, biometric, brainwave, and body data and extract emotions to provide emotional application services. Emotional application service technology is evolving, and exhibits significant potential for use in a variety of fields, such as entertainment, healthcare, market analysis, online education, automobiles, customer marketing, and general home use.

2.4. Stress and Bio-Information. Previous studies have used biosignals as a method of identifying stress states in individuals. Bakker et al. [16], Healey and Picard [17], and Jung and Yoon [18] have collected biosignals from workers, drivers, and senior citizens, respectively, to evaluate stress levels. Setz et al. [19], Melillo et al. [20], and Kurniawan et al. [21] administered tests that required learning capabilities to create experimental environments, and then identified stress states. However, these previous studies have been limited by the fact that they considered the identification of a posteriori states, and did not consider combinations of features that are appropriate for stress relief using emotions. In this chapter, previous studies on the identification of stress states using biosignals are listed by their purpose, the biosignals that they used, and their analysis techniques, as shown in Table 1. Previous studies have identified stress states using various combinations of biosignals such as HR, GSR, ECG, EMG, and brainwaves. Of these, the heartbeat data has been used most often to identify stress. However, because heartbeat variability can occur in a variety of contexts other than stress, this is used in combination with other data [22]. Sun et al. [23] used the heartbeat data along with GSR and accelerometer data to identify stress during physical activities. However, because GSR is more sensitive to movement than other biosignals, it is difficult to obtain accurate data in situations with a lot of movement. Kurniawan et al. performed experiments using voice data to analyze the words uttered by subjects and identify stress [21]. In studies by Setz et al. and Melillo et al., subjects were given tests that required learning capabilities to create experimental environments. In this manner, previous studies have faced difficulties in adequately measuring and analyzing user stress without using a variety of biometrics. In addition, the goals of studies on stress that have used biometrics have only been focused on measurement and analysis. As such, there has been a lack of studies on stress and emotions that are measured via biometrics. This study considers the limitations of previous work, and employs user biometrics such as brainwaves, pulse, and blood pressure (systolic and diastolic blood pressure). In addition, it proposes a system that uses biosignals to identify a user's stress states and infer emotions, to suggest colors and sounds according to the user's emotional state, i.e., their stress index.

\section{System Configuration and Design}

To infer and classify the emotional state of a user according to the user's stress, this study designed a fuzzy system by using the EEG data after acquiring the EEG, pulse rate, and blood pressure from the sensors, and the pulse rate and blood pressure (systolic and diastolic blood pressure) data were produced in single packets separately and sent to the database. The SVM 
TABLE 1: Summary of related studies.

\begin{tabular}{|c|c|c|c|}
\hline Objects (topics) & Signals used & Analysis methodologies & References \\
\hline $\begin{array}{l}\text { Automatic identification of stress causes } \\
\text { of employees }\end{array}$ & GSR & Adaptive windowing & Bakker et al. [16] \\
\hline Detecting real-world driving stress & HR, EMG, Respiration & Continuous correlations & Healey and Picard [17] \\
\hline $\begin{array}{l}\text { Multi-level assessment model for } \\
\text { monitoring elder's health condition }\end{array}$ & HR, EEG, ECG & SVM, DT, Expectation maximization & Jung and Yoon [18] \\
\hline $\begin{array}{l}\text { Personal health system for detecting } \\
\text { stress }\end{array}$ & GSR & Latent dirichlet allocation, SVM & Setz et al. [19] \\
\hline \multirow{2}{*}{ Stress elicitation by examination } & HR & Latent dirichlet allocation & Melillo et al. [20] \\
\hline & Voice, GSR & DT, SVM, K-Means & Kurniawan [21] \\
\hline $\begin{array}{l}\text { Activity-aware mental stress detection } \\
\text { (sitting, standing and walking) }\end{array}$ & HR, GSR, Accelemeter & DT, SVM, Bayes network & Sun et al. [23] \\
\hline $\begin{array}{l}\text { Automatic cry detection in early } \\
\text { childhood }\end{array}$ & Voice & Gentle-boost & Ruvolo and Movellan [24] \\
\hline $\begin{array}{l}\text { Automatic classification of infant crying } \\
\text { for early disease detection }\end{array}$ & Voice & Genetic selection of a fuzzy model & Rosales-Pérez et al. [25] \\
\hline $\begin{array}{l}\text { Automatic detection of the expiratory } \\
\text { and inspiratory phases in newborn cry } \\
\text { signal }\end{array}$ & Voice & Hidden markov model & Abou-Abbas et al. [26] \\
\hline
\end{tabular}

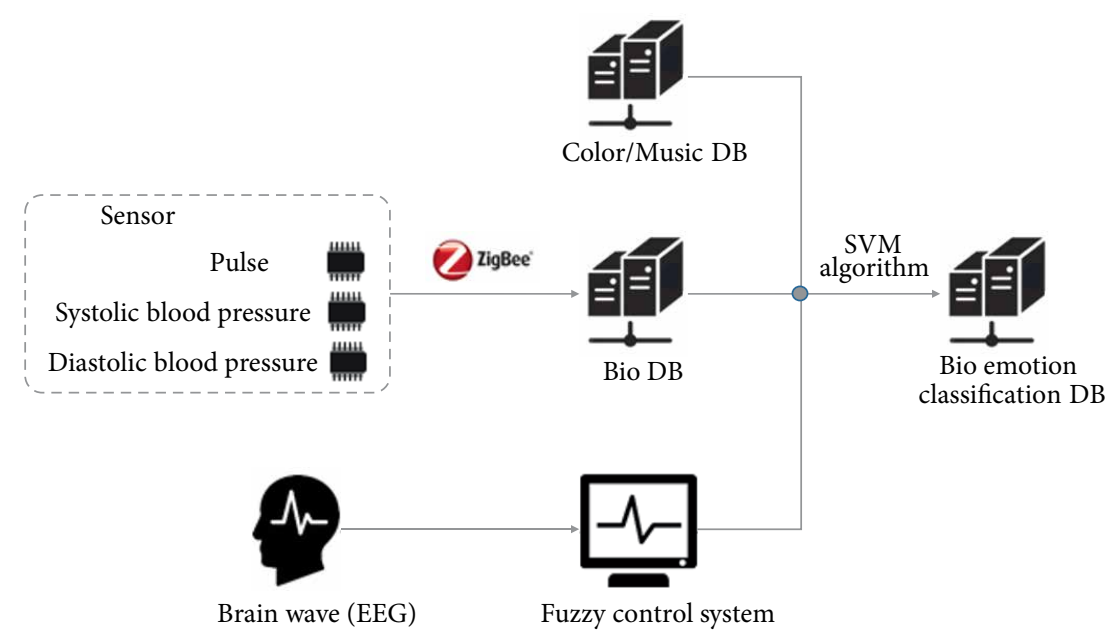

FIGURE 1: The system configuration diagram.

algorithm was used to classify the biometric emotion information according to the stress index by using the acquired EEG, pulse rate, and blood pressure data of the fuzzy control system. The biometric emotion information is classified into color values corresponding to the emotion based on 20 color emotion models selected through HP's "the Meaning of Color," and as for the music, the music pieces provided by "Samsung Idea" for music therapy were collected, classified, and used [27]. Figure 1 shows the configuration diagram of the system proposed in this paper, which performs emotional classification and recommendation based on biometrics and stress index.

This study designed a fuzzy system to classify the biometric emotion information according to a user's stress index by using the EEG data. The stress emotion information expressed by the fuzzy control system can be divided into four types: stable, relaxed, tensed, and excited. BIOPAC MP 150 was used to measure the EEG of the user and the measurement was conducted according to the 10-20 International System of Electrode Placement $[28,29]$. When conducting the measurement, sampling was performed at $256 \mathrm{~Hz}$ for only the EEG data that passed through a notch filter of $60 \mathrm{~Hz}$ in terms of hardware. The sampled data were filtered in the frequency band $0.5-50 \mathrm{~Hz}$. To analyze the EEG data, the data for $60 \mathrm{~s}$, which is considered the stable-state analysis section for the analysis of EEG, were used excluding each 30 s part of starting and ending. First, the EEG data, which are the data in time domain, were converted into frequency domain by using fast Fourier transform, as shown in Equation (1) in order to analyze them.

$$
H\left(f_{n}\right)=\sum_{k=0}^{N-1} h_{k} e^{-j 2 \pi k n / N} .
$$

To extract an absolute size by each frequency, the data were converted into the frequency domain through the Fourier 


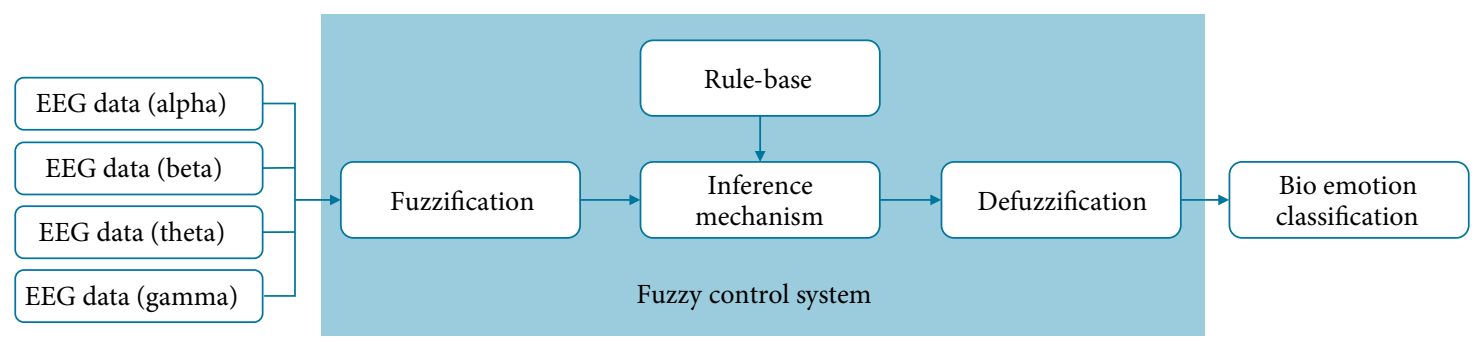

FIGURE 2: The fuzzy control system using EEG data based on bio emotion classification.

transform process. Moreover, the power spectrum analysis method was used for comparison and analysis [30]. The power spectrum analysis method is a widely used analysis method in many areas including biosignals. Depending on the expression method, it is divided into one-side and two-side. One-side shows 0 and positive frequency domain only, and two-side shows the frequency domain of \{negative, 0 , positive\}. This study obtained the absolute values of power spectrum for the band frequencies of $\alpha, \beta, \theta$, and $\gamma$ by using the one-side power spectrum analysis method. The Fourier inverse transform is shown in Eq. (2)

$$
h_{k}=\frac{1}{N} \sum_{k=0}^{N-1} H_{n} e^{-j 2 \pi k n / N} .
$$

After obtaining the absolute values on both sides of Equation (2), if they are squared and added, then Equation (3) is induced. Here, it can be confirmed that the sum of square of the signal that was subjected to the Fourier transform is the same as the sum of square of the original signal. Here, the sum of Fourier transform or the sum of square of the original signal is called the total power value. In other words, it indicates that the total power value is identical in both the frequency space and the temporal space. This is called Parseval theorem [31].

$$
\text { Total Power }=\sum_{k=0}^{N-1}\left|h_{k}\right|^{2}=\frac{1}{N} \sum_{n=0}^{N-1}\left|H_{n}\right|^{2}
$$

The one-side spectrum analysis method that satisfies the Parseval theorem is shown in

$$
\begin{gathered}
P\left(f_{0}\right)=P(0)=\frac{1}{N^{2}}\left|H_{0}\right|^{2}, \\
P\left(f_{0}\right)=\frac{1}{N^{2}}\left[\left|H_{n}\right|^{2}+\left|H_{N-n}\right|^{2}\right], \quad n=1,2, \ldots,\left(\frac{N}{2}-1\right), \\
P\left(f_{n / 2}\right)=P\left(f_{c}\right)=\frac{1}{N^{2}}\left|H_{N / 2}\right|^{2} .
\end{gathered}
$$

This study extracted the absolute values of the power spectrum of theta $(4-8 \mathrm{~Hz})$, alpha $(8-14 \mathrm{~Hz})$, beta $(14-30 \mathrm{~Hz})$, and gamma $(30-50 \mathrm{~Hz})$ domains through the one-side power spectrum analysis, and used it as an input value of the fuzzy control system to classify the emotion.
TABle 2: The fuzzy condition using EEG data.

If theta is high and alpha, beta, gamma is low then emotion is stability

If alpha is high, and beta, theta, gamma is low then emotion is relaxation

If beta is high and alpha, theta, gamma is low then emotion is tension

If gamma is high and alpha, beta, theta is low then emotion is excitement

To classify the emotion of the user, the fuzzy model should be constructed by quantifying the physical information that can identify the emotion of the user from the EEG data. To this end, the correlation of $\alpha, \beta, \theta$, and $\gamma$ values, i.e., the absolute power values acquired from the user with the user emotion, was investigated to define the rules. Then, the fuzzy model was implemented as shown in Figure 2. In other words, the biometric emotion classification (stability, relaxation, tension, and excitement) was performed by applying the four fuzzy rules using the absolute power values $\alpha, \beta, \theta$, and $\gamma$ obtained from the EEG data of the user as the input values. The fuzzy control rules are expressed as linguistic control rules of "IF Ai THEN Bi" format. To create a set of control rules, multiple rules are collected and by making an inference based on the rules, an output value is obtained. Table 2 shows the fuzzy condition for expressing the output value according to the input variable by using the EEG data.

Defuzzification refers to a process of converting a fuzzy quantity result, which is obtained from the fuzzy inference, to a representative value. In this study, the center of gravity method was used for the fuzzy inference $[32,33]$. Equation (5) shows the inference output function defuzzificated by the four fuzzy rules.

$$
C^{\prime}=\frac{\sum_{i} b_{i} \int \mu c_{i}^{\prime}(z)}{\sum_{i} \int \mu c_{i}^{\prime}(z)}
$$

To measure the pulse rate and blood pressure data of the user, this study used the ZigBee wireless sensor network. ZigBee of IEEE 802.15.4 refers to a short-range wireless communication technology that focuses on applications requiring low power consumption, low speed, and low cost [34]. A sensor module of integrating pulse rate and blood pressure sensors was used to acquire the data. Furthermore, the data were measured by using a Telos platform series as the process board, MSP430 MCU, and CC256XQFNEM. If the pulse rate and blood pressure data are produced in single packets separately and used, 


\begin{tabular}{|c|c|c|c|c|c|c|c|c|c|c|}
\hline $7 \mathrm{E} \quad 00$ & $0 \mathrm{~A}$ & $7 \mathrm{D}$ & 10 & $00 \quad 00$ & $02 \quad 00$ & 00 & $01 \quad 00$ & $02 \quad 00$ & $\begin{array}{llll}\mathrm{EE} & \mathrm{D} 3 & \mathrm{FF} & \mathrm{FF}\end{array}$ & $55 \quad 00$ \\
\hline 1 & 2 & 3 & 4 & 5 & 6 & 7 & 8 & 9 & 10 & 11 \\
\hline 7E 00 & $0 \mathrm{~A}$ & $7 \mathrm{D}$ & 10 & 0000 & 0200 & 0000 & 0100 & 0200 & EE D3 FF FF & 5500 \\
\hline \multicolumn{4}{|c|}{$\begin{array}{l}\text { 1: Address 2: MSG } \\
\text { 7: Sequence number }\end{array}$} & \multicolumn{2}{|c|}{$\begin{array}{l}\text { 3: GroupID } \\
\text { 8: Hop count }\end{array}$} & $\begin{array}{l}\text { 4: Data l } \\
\text { 9: Ad }\end{array}$ & & \multicolumn{2}{|c|}{$\begin{array}{l}\text { 5: Source address } \\
\text { 10: Time stamp }\end{array}$} & 6: Origin address \\
\hline
\end{tabular}

FIGURE 3: The sensed sensor data structure.

energy is consumed based on additional traffic and data transmission. Therefore, they were bundled in one packet and then sent to the database. Figure 3 shows the structure of sensed biometric data. MSG shows the type of biometric information and enables to isolate the type of pulse rate, i.e., systolic or diastolic blood pressure. GroupID shows the sensor information and each sensor has a GroupID. Timestamp is the time when the sensor measured the data. Reading expresses the actually measured data values in hexadecimal 2 bytes.

Various learning algorithms are used to match the biometric emotion information according to the stress index. Nevertheless, this study used the SVM algorithm, which can solve the nonlinear discrimination problem of multilayer perceptron structure because the EEG, pulse rate, and blood pressure data used in this study consist of nonlinear data structures. The goal of the SVM algorithm is to find a classification boundary by maximizing the margin between the closest observation values (support vectors) in two classes. Furthermore, even when linear separation is difficult, classification is facilitated by using a nonlinear hyperplane through a kernel function, and consequently, it is used in diverse areas such as biology, and image and text recognition. To optimize the performance level of learning data, most traditional pattern recognition methods are based on a risk minimization method. In the case of the SVM algorithm, it is based on a structural risk method to minimize the probability of incorrect classification of the data having fixed but unknown probability distribution [35]. When data cannot be linearly separated like the data of this study, i.e., when they have a pattern that cannot be completely separated because they are overlapped with each other at the linear separation boundary, a slack variable $\xi_{i}$ is used considering a case of incorrect classification, as shown in eqs. (6) and (7)

$$
\begin{aligned}
& \text { minimize }: J(w, \xi)=\frac{1}{2}|w|^{2}+C \sum_{i=1}^{N} \xi_{i}, \\
& \text { subject to }:\left\{\begin{array}{l}
y_{i}\left(w^{T} x_{i}+b\right) \geq 1-\xi_{i}, \forall i, \\
\xi_{i} \geq 0, \forall i,
\end{array}\right.
\end{aligned}
$$

However, $C$ is a trade-off parameter and the Lagrangian function is expressed by

$$
\begin{aligned}
J(w, b, \alpha)= & \frac{1}{2} w^{T} w+C \sum_{i=1}^{N} \xi_{i} \\
& -\sum_{i=1}^{N} \alpha_{i}\left(y_{i}\left(w^{T} x_{i}+b\right)-1+\xi_{i}\right)-\sum_{i=1}^{N} \mu_{i} \xi_{i} .
\end{aligned}
$$

TABLE 3: The SVM algorithm configuration.

Algorithm: SVM
Number of data for learning: $N$
Inputs: sample $x$ to classify dataset : $I_{i}$
$I_{i 1}:$ EEG, $I_{i 2}:$ Pulse, $I_{i 3}:$ Systolic Blood Pressure,
$I_{i 4}:$ Diastolic Blood Pressure
Output : decision $y \in\{-1,1\}$
Classify using SVM Algorithm, get the result in the form of a real
number.

The above Equation (8) can be converted to Equations (9) and (10) by applying a Lagrangian optimization method.

$$
\begin{aligned}
& \text { minimize }: \sum_{i=1}^{N} \alpha_{i}-\frac{1}{2} \sum_{i=1}^{N} \sum_{i=j}^{N} \alpha_{i} \alpha_{j} y_{i} y_{j} x_{i}^{T} x_{j}, \\
& \text { subject to : }\left\{\begin{array}{l}
\sum_{i=1}^{N} \alpha_{i} y_{i}=0, \\
0 \leq \alpha_{i} \leq C, \quad \forall i=1, \ldots, N,
\end{array}\right.
\end{aligned}
$$

If the above equations are solved, a decision function can be obtained, as shown in Eq. (11)

$$
f(x)=\operatorname{sgn}\left(\sum_{i=1}^{N} y_{i} \alpha_{i}\left(x_{i}^{T} x\right)+b\right) .
$$

The SVM algorithm developed for binary classification has many difficulties when solving problems having many classes in a real environment, one-against-all and one-against-one methods have been proposed. Among them, the one-againstone method consists of $k(k-1) / 2$ quantity of the SVM algorithm when $k$ classes are inputted. Furthermore, for respective learning data that consist of data showing two groups they belong to, as the number of learning data used is small when performing the learning, the learning speed is fast $[35,36]$. Therefore, this study conducted experiments by using the oneagainst-one method to improve the learning performance and composed the SVM algorithm as shown in Table 3.

As various kinds of emotions can be expressed according to the external environment, it is effective to predefine the emotion colors and music to be used. Accordingly, for the classification of emotion colors, this study selected 20 color emotion models as the representative elements from HP's "the Meaning of Color." Through HP's color table and the emotion vocabulary matching result investigated in this study, the 
TABLE 4: The common emotional words analysis according to color.

\begin{tabular}{|c|c|c|c|}
\hline Color & Meaning & Color & Meaning \\
\hline \multirow{2}{*}{ Bright red } & $\begin{array}{l}\text { Optimistic, dynamic, energizing, exciting, sexy, intense, } \\
\text { stimulating, aggressive, powerful, energetic, dangerous }\end{array}$ & \multirow{2}{*}{ Orange } & $\begin{array}{l}\text { Ambition, fun, happy, energetic, balance, } \\
\text { flamboyant, warmth, enthusiasm, generosity, } \\
\text { vibrant, expansive, organic }\end{array}$ \\
\hline & $\begin{array}{l}\text { International significance: } \text { China = good luck; } \\
\text { India = purity; Eastern cultures = signifies joy when } \\
\text { combined with white }\end{array}$ & & $\begin{array}{l}\text { International significance: Ireland = religious } \\
\text { significance(protestant) }\end{array}$ \\
\hline Burgundy & $\begin{array}{c}\text { Vigor, elegance, richness, refinement, leadership, maturity, } \\
\text { expensive }\end{array}$ & Light blue & $\begin{array}{c}\text { Peace, tranquillity, quiet, cool, clean, soft, pure, } \\
\text { understanding }\end{array}$ \\
\hline \multirow{2}{*}{ Blue } & $\begin{array}{l}\text { Truth, healing, tranquility, stability, peace, harmony, } \\
\text { wisdom, trust, calm, confidence, protection, security, } \\
\text { loyalty }\end{array}$ & Purple & $\begin{array}{l}\text { Spirituality, royalty, mystery, wisdom, } \\
\text { transformation, independence, enlightenment, } \\
\text { respect, wealth }\end{array}$ \\
\hline & $\begin{array}{l}\text { International significance: } \text { China }=\text { immortality; } \\
\text { Hindus }=\text { color of Krishna }\end{array}$ & Navy & $\begin{array}{l}\text { Dignity, credibility, strength, authority, } \\
\text { conservative, trustworthiness, traditional, quiet, } \\
\text { confident, serene }\end{array}$ \\
\hline \multirow{3}{*}{ Green } & $\begin{array}{l}\text { Nature, envy, healing, fertility, good luck, hope, stability, } \\
\text { success, generosity }\end{array}$ & Beige & $\begin{array}{l}\text { Earthy, classic, neutral, warm, soft, bland, } \\
\text { melancholy }\end{array}$ \\
\hline & $\begin{array}{l}\text { International significance: China \& France = negative } \\
\text { significance for package goods; India = color of Islam; some }\end{array}$ & $\begin{array}{l}\text { Greenish } \\
\text { yellow }\end{array}$ & Tart, fruity, acidy, jealousy \\
\hline & tropical countries $=$ danger & Lime & Tart, fruity, acidy, refreshing, lively, revitalizing \\
\hline \multirow{2}{*}{ Brown } & $\begin{array}{l}\text { Stability, masculinity, reliability, comfort, endurance, } \\
\text { simplicity, friendship }\end{array}$ & Terracotta & $\begin{array}{l}\text { Wholesome, earthy, country, welcoming, } \\
\text { warmth, stability, fall, harvest }\end{array}$ \\
\hline & $\begin{array}{l}\text { International significance: } \text { Colombia }=\text { discourages sales; } \\
\text { India }=\text { the color of mourning }\end{array}$ & Lavender & $\begin{array}{c}\text { Enchantment, nostalgia, delicacy, floral, sweet, } \\
\text { fashion }\end{array}$ \\
\hline Light pink & $\begin{array}{l}\text { Love, romance, softness, delicacy, sweetness, friendship, } \\
\text { tenderness, fidelity, compassion }\end{array}$ & Teal blue & $\begin{array}{c}\text { Emotional healing, pleasing, rich, protection, } \\
\text { unique, expensive }\end{array}$ \\
\hline $\begin{array}{l}\text { Bright } \\
\text { yellow }\end{array}$ & $\begin{array}{l}\text { Cheeriness, joy, action, optimism, happiness, idealism, } \\
\text { summer, hope, imagination, sunshine, philosophy, youth, } \\
\text { International significance: Asia = sacred, imperial }\end{array}$ & Olive green & $\begin{array}{c}\text { Traditional color of peace, camouflage, classic, } \\
\text { adventure } \\
\text { International significance: Military }\end{array}$ \\
\hline Fuchsia & Hot, sensual, exciting, bright, fun, energetic, feminine & Neural gray & $\begin{array}{l}\text { Neutral, corporate, classic, practical, cool, } \\
\text { timeless, quiet, quality }\end{array}$ \\
\hline
\end{tabular}

classification was performed based on the common emotion vocabulary, as shown in Table 4.

This study classified the colors and music corresponding to the biometric emotion information according to the stress index by using wavelength, which is a common characteristic of emotion information, color, and sound. As wavelength and frequency have an inversely proportional relationship in physics and mathematics, they can be converted to each other mathematically. Based on $\mathrm{C}$ (on the musical scale), the relationship with the wavelength ratio of $\mathrm{D}$ and $\mathrm{E}$ is $1: 4 / 5: 2 / 3$. This ratio is consistent with the ratio of the respective wavelength of three primary colors, i.e., red, green, and blue: $650 \mathrm{~nm}, 520 \mathrm{~nm}$, and $433 \mathrm{~nm}$. As such, C, D, and E are similar to the three primary colors, which can be used to create countless colors through appropriate mixing. Therefore, if the wavelength ratio of dodecatonic scale based on the equal temperament is corresponded sequentially to the frequency of colors that can be created with the combination of the three primary colors, the colors and sounds can be linked [37].

By analyzing the color measurement values and the musical scale of music, the optimal colors and list of music corresponding to the biometric emotion information are determined according to the stress index. Music, which is an important emotion information for understanding and analyzing the emotions, can heal the emotion of the user and relieve stress. Therefore, to recommend music based on the biometric emotional state according to the stress index, this study composed a music list based on the data collected from "Samsung Idea" for musical therapy. Table 5 classifies the biometric emotion information according to the stress index and shows the classification of the corresponding colors and music.

\section{Performance Evaluation and Experiment Results}

In this study, the experiments were conducted by using the EEG, pulse rate, and blood pressure as input data to classify the biometric emotion information according to the stress index. Table 6 classifies the four states (stability, relaxation, tension, and excitement) based on the classification standard of biometric emotion information according to the stress index $[38,39]$.

This study used the SVM algorithm to design and evaluate the classification of respective emotions. The SVM algorithm is a classification algorithm that determines the discrimination boundary in order to have the largest distance between the discrimination boundary and each class. The kernel of the SVM algorithm used in this study was the radial basis function 
TABLE 5: The corresponding emotional colors and corresponding emotional music according to stress index.

\begin{tabular}{lccc}
\hline Stress index & $\begin{array}{c}\text { Bio emotion } \\
\text { information }\end{array}$ & $\begin{array}{c}\text { Corresponding } \\
\text { emotion colors }\end{array}$ & Corresponding emotion music \\
\hline Stage $1(00-30)$ & Stability & Yellow, Brown & Franz Peter Schubert-10 songs besides the lullabies \\
Stage 2 $(31-60)$ & Relaxation & Red & Antonio Vivaldi-10 other songs besides the four seasons (spring) \\
Stage $3(61-70)$ & Tension & Blue & Franz Peter Schubert-10 songs besides the Ave Maria \\
Stage $4(71-100)$ & Excitement & Green & Robert Alexander Schumann-10 songs besides the dream \\
\hline
\end{tabular}

TABLE 6: The classification of bio emotion information according to stress index.

\begin{tabular}{|c|c|c|c|c|c|}
\hline \multirow{2}{*}{ Stress index } & \multirow{2}{*}{ Bio emotion information } & \multirow{2}{*}{$\begin{array}{c}\text { EEG } \\
\mathrm{Hz}\end{array}$} & \multirow{2}{*}{$\begin{array}{c}\text { Pulse } \\
\text { Times/Minute }\end{array}$} & \multicolumn{2}{|c|}{ Blood pressure } \\
\hline & & & & Diastolic & Systolic \\
\hline Stage 1 & Stability & Theta (4-7) & $70-80$ & 80 & 120 \\
\hline Stage 2 & Relaxation & Alpha (8-14) & $60-70$ & $81-89$ & $121-139$ \\
\hline Stage 3 & Tension & Beta $(14-30)$ & $80-90$ & $90-99$ & $140-159$ \\
\hline Stage 4 & Excitement & Gamma (30-50) & Under 60, Over 90 & Over 100 & Over 160 \\
\hline
\end{tabular}

TABLE 7: The confusion matrix.

\begin{tabular}{lccccc}
\hline \multirow{2}{*}{ Type } & & \multicolumn{3}{c}{ True condition } \\
& & Stability & Relaxation & Tension & Excitement \\
\hline \multirow{3}{*}{ Prediction condition } & Stability & 90.4 & 11.4 & 0.0 & 0.0 \\
& Relaxation & 9.6 & 83.4 & 3.4 & 0.5 \\
& Tension & 0.0 & 5.2 & 0.0 & 13.8 \\
\hline
\end{tabular}

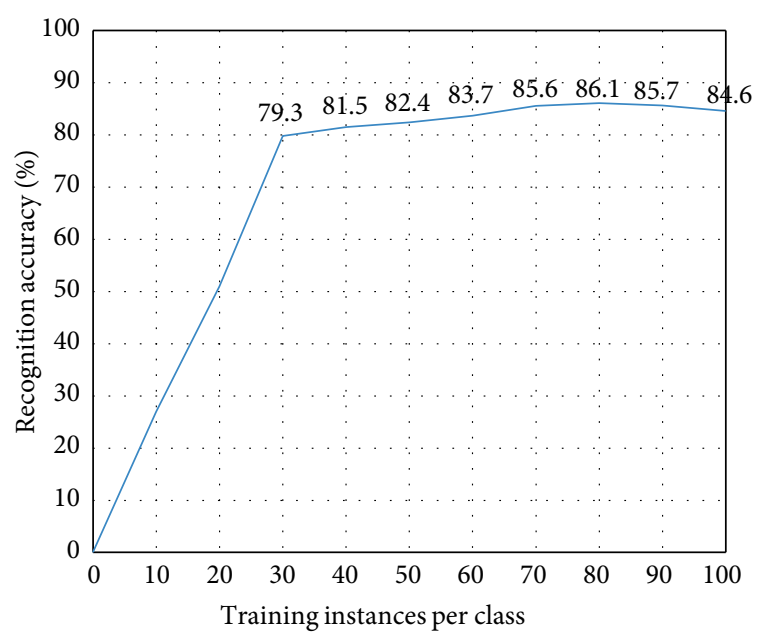

FIGURE 4: The bio emotion classification accuracy.

kernel; the radius of the kernel was set to 1 and the margin of the SVM algorithm was set to $1[40,41]$. Figure 4 shows the accuracy result of classifying according to the four types of biometric emotion information through the SVM algorithm. When $80 \%$ of data were learned according to the ratios of learning data, the highest performance of $86.1 \%$ was shown. Furthermore, Table 7 shows the error matrix when $80 \%$ of data were learned. The classification results were $90.4 \%$ for stable, $83.4 \%$ for relaxed, $84.5 \%$ for tensed, and $85.7 \%$ for excited.

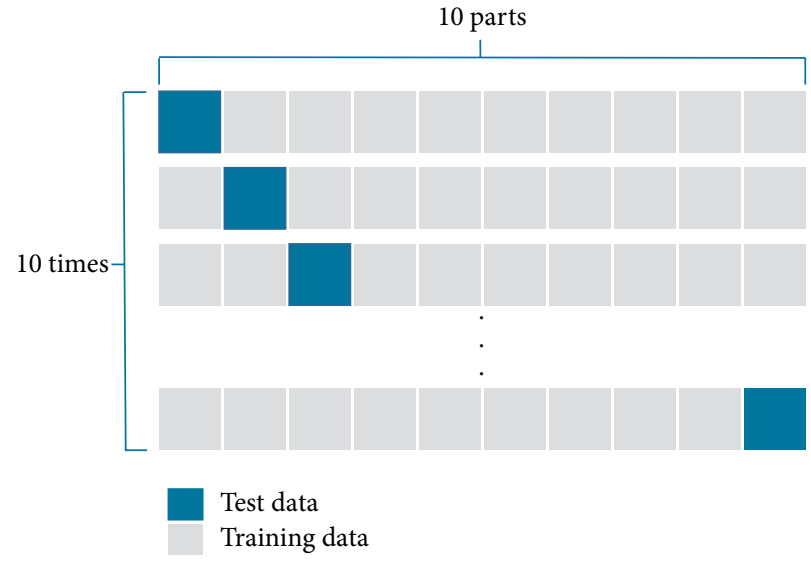

FIGURE 5: The 10-fold cross validation.

Furthermore, to evaluate the tracking performance of the proposed system that recommends colors and music according to the biometric emotion information and stress index, 10 -fold cross validation was conducted, thereby minimizing the influence of training data and ensuring reliability. The 10 -fold cross validation divides the entire data into ten equal parts and uses nine parts as training data and the remaining part as test data. The 10 -fold cross validation repeats the training and testing ten times in total to evaluate the overall performance, and every time, one equal part of data used for the test is changed. Figure 5 shows an example of 10 -fold cross validation. 
TABLE 8: The evaluation of data performance for verification.

\begin{tabular}{lc}
\hline Fold no. & Accuracy (unit: \%) \\
\hline 1 & 84.8 \\
2 & 87.5 \\
3 & 88.6 \\
4 & 86.4 \\
5 & 87.3 \\
6 & 83.3 \\
7 & 89.6 \\
8 & 87.4 \\
9 & 83.2 \\
10 & 85.6 \\
\hline
\end{tabular}

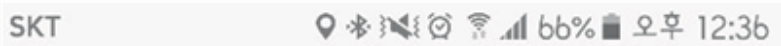 \\ Bio Emotion Classification System}

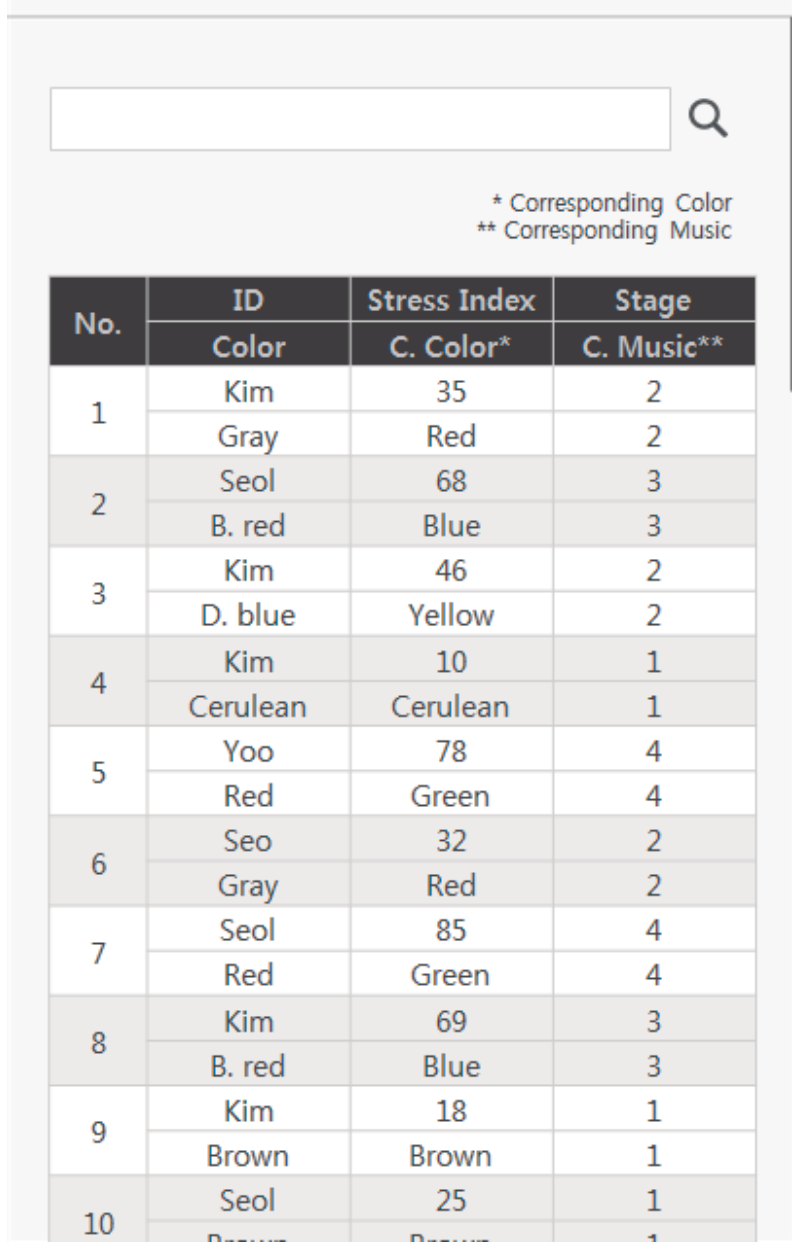

FIGURE 6: The corresponding emotion color and corresponding emotion music recommendation system according to bio emotion information and stress index.

This study divided the experimental data into evaluation data and validation data using the ratio $7: 3$. Furthermore, the system was optimized by using the leave-one-out method.
Table 8 shows the average accuracy of $86.4 \%$ in the result of validation data obtained through the performance evaluation. Therefore, the performance of the recommendation system is high.

Figure 6 shows the result of implementing an application for the proposed system that recommends the colors and music according to the biometric emotion information and stress index. The emotion colors and the emotion music are classified into the corresponding items according to the biometric emotion information and stress index.

\section{Conclusion}

This study aimed to classify and recommend the emotion colors and the emotion music corresponding to the emotion of the user by measuring biometric information. Accordingly, after measuring the EEG, pulse rate, and blood pressure data (i.e., the biometric information), the emotion colors and emotion music are classified according to the current biometric emotion information and stress index of the user through real-time emotion analysis. To classify the EEG data into four types of biometric emotion information (stability, relaxation, tension, and excitement), a fuzzy control system was designed and the pulse rate and blood pressure data were composed in single packets separately and sent to the database. When $80 \%$ of data were learned according to the ratio of the training data by using the SVM algorithm to classify the EEG, blood pressure, and pulse rate data based on the biometric emotion information, the highest performance of $86.1 \%$ was shown. Moreover, the error matrix classification results obtained were $90.4 \%$ for stable, $83.4 \%$ for relaxed, $84.5 \%$ for tensed, and $85.7 \%$ for excited, confirming that the efficiency was high. Furthermore, when the 10 -fold cross validation was performed to evaluate the tracking performance of the system that recommends the emotion colors and emotion music according to the biometric emotion information and stress index, an average accuracy of $86.4 \%$ was demonstrated, confirming that the performance of the recommendation system was high. This study proposed a biometric emotion information classification system using the stress index to classify the emotion colors and emotion music intelligently based on the emotion of the user. Therefore, it is expected to contribute to studies on $\mathrm{HCI}$ in the fourth industrial revolution era. In a future study, the emotions will be classified based on the situational and environmental factors as well as the biometric information of the user. Furthermore, the accuracy and efficiency of the proposed system will be enhanced by using various types of biometric information in addition to the EEG, pulse rate, and blood pressure used in this study, as measures for determining biometric emotions.

\section{Data Availability}

The BIOPAC MP 150 EEG data used to support the findings of this study have been deposited in the BrainAmp by Brain Products, Munich, Germany. 


\section{Conflicts of Interest}

The authors declare that they have no conflicts of interest regarding the publication of this paper.

\section{Acknowledgments}

This research was supported by the National Research Foundation of Korea (NRF) grant funded by the Korea Government (MSIT) (No. 2019R1F1A1041186). This research was supported by the Korean MSIT (Ministry of Science and ICT), under the National Program for Excellence in SW (20170-00137), supervised by the IITP (Institute for Information \& communications Technology Planning \& Evaluation).

\section{References}

[1] A. Y. Kim, E. H. Jang, and J. H. Sohn, "Classification of negative emotions based on arousal score and physiological signals using neural network," Korean Society for Emotion and Sensibility, vol. 21, no. 1, pp. 177-186, 2018.

[2] L. Santamaria-Granados, M. Munoz-Organero, G. RamirezGonzalez, E. Abdulhay, and N. Arunkumar, "Using deep convolutional neural network for emotion detection on a physiological signals dataset (AMIGOS)," IEEE Access, vol. 7, pp. 57-67, 2017.

[3] R. W. Picard, “Affective computing: challenges," International Journal of Human-Computer Studies, vol. 59, no. 1-2, pp. 55-64, 2003.

[4] S. M. Park, Y. H. Lee, K. E. Ko, and K. B. Sim, "Development of EEG signals measurement and analysis method based on timbre," International Journal of Fuzzy Logic and Intelligent Systems, vol. 20, no. 3, pp. 388-393, 2010.

[5] W. Apriadi, S. K. Wijaya, and L. O. H. Prawito Zilullah, "Development of electroencephalogram (EEG) based on ADS1 299EEGFE-PDK and LaunchPad MSP432P401R," in 2017 5th International Conference on Instrumentation, Communications, Information Technology, and Biomedical Engineering (ICICI$B M E)$, IEEE, Indonesia, 2017.

[6] Y. H. Cho and K. S. Park, "A study on the improvement of emotion recognition by gender discrimination," Journal of the Institute of Electronics Engineers of Korea, vol. 45, no. 4, pp. 107-114, 2008.

[7] M. V. Villarejo, B. G. Zapirain, and A. M. Zorrilla, "Algorithms based on CWT and classifiers to control cardiac alterations and stress using an ECG and a SCR," Sensors (Basel, Switzerland), vol. 13, no. 5, pp. 6141-6170, 2013.

[8] M. R. Ogiela and L. Ogiela, "On using cognitive models in cryptography," in 2016 IEEE 30th International Conference on Advanced Information Networking and Applications (AINA), Institute of Electrical and Electronics Engineers, pp. 1055-1058, Crans-Montana, Switzerland, 2016.

[9] L. Ogiela and M. R. Ogiela, "Insider threats and cryptographic techniques in secure information management," IEEE Systems Journal, vol. 11, no. 2, pp. 405-14, 2017.

[10] N. Mitsuo, "Kansei engineering: a new ergonomic consumeroriented technology for product development," International Journal of Industrial Ergonomics, vol. 15, no. 1, pp. 3-11, 1995.
[11] W. Hongwei and M. M. Jerry, "Classification of battlefield ground vehicles using acoustic features and fuzzy logic rulebased classifiers," IEEE Transactions on Fuzzy Systems, vol. 10, no. 1, pp. 56-72, 2007.

[12] J. Nicholson, K. Takahashi, and R. Nakatsu, "Emotion recognition in speech using neural networks," Neural Computing and Applications, vol. 9, no. 4, pp. 290-296, 2000.

[13] C. C. Lee, E. Mower, C. Busso, S. Lee, and S. Narayanan, "Emotion recognition using a hierarchical binary decision tree approach," Speech Communication, vol. 53, no. 9-10, pp. 1162-1171, 2011.

[14] K. E. Ko, H. C. Yang, and K. B. Sim, "Emotion recognition using EEG signals with relative power values and Bayesian network," International Journal of control Automation and Systems, vol. 7, no. 5, pp. 865-70, 2009.

[15] V. Shusterman and O. Barnea, "Sympathetic nervous system activity in stress and biofeedback relaxation," IEEE Engineering in Medicine and Biology Magazine, vol. 24, no. 2, pp. 52-57, 2005.

[16] J. Bakker, M. Pechenizkiy, and N. Sidorova, "What's your current stress level? Detection of stress patterns from GSR sensor data," in Proceedings of the IEEE International Conference on Data Mining Workshop, pp. 573-580, IEEE, 2011.

[17] J. A. Healey and R. W. Picard, "Detecting stress during real-world driving tasks using physiological sensors," IEEE Transactions on Intelligent Transportation Systems, vol. 6, no. 2, pp. 156-66, 2005.

[18] Y. C. Jung and Y. I. Yoon, "Monitoring senior wellness status using multimodal biosensors," in Proceedings of the 2016 International Conference on Big Data and Smart Computing (BigComp), pp. 435-438, IEEE, Hong Kong, China, 2016.

[19] C. Setz, B. Arnrich, J. Schumm, R. La Marca, G. Tröster, and U. Ehlert, "Discriminating stress from cognitive load using a wearable EDA device," IEEE Transactions on Information Technology in Biomedicine, vol. 14, no. 2, pp. 410-417, 2010.

[20] P. Melillo, M. Bracale, and L. Pecchia, "Nonlinear heart rate variability features for real-life stress detection. Case study: students under stress due to university examination," BioMedical Engineering OnLine, vol. 10, no. 1, p. 96, 2011.

[21] H. Kurniawan, A. V. Maslov, and M. Pechenizkiy, "vStress detection from speech and galvanic skin response signals," in Proceedings of the 26th IEEE International Symposium on Computer Based Medical Systems, pp. 209-214, IEEE, 2013.

[22] N. Sharma and T. Gedeon, "Objective measures, sensors and computational techniques for stress recognition and classification: a survey," Computer Methods and Programs in Biomedicine, vol. 108, no. 3, pp. 1287-1301, 2012.

[23] F. T. Sun, C. Kuo, H. T. Cheng, S. Buthpitiya, P. Collins, and M. Griss, "Activity-aware mental stress detection using physiological sensors," in Proceedings of the International Conference on Mobile Computing, Applications, and Services, pp. 211-230, Springer, 2010.

[24] P. Ruvolo and J. Movellan, "Automatic cry detection in early childhood education settings," vol. 7, in Proceedings of the IEEE International Conference on Development and Learning, IEEE, pp. 204-208, Monterey, CA, USA, 2008.

[25] A. Rosales-Pérez, C. A. Reyes-García, J. A. Gonzalez, O. F. ReyesGalaviz, H. J. Escalante, and S. Orlandi, "Classifying infant cry patterns by the genetic selection of a fuzzy model," Biomedical Signal Processing and Control, vol. 17, pp. 38-46, 2015.

[26] L. Abou-Abbas, H. F. Alaie, and C. Tadj, "Automatic detection of the expiratory and inspiratory phases in newborn cry signals," 
Biomedical Signal Processing and Control, vol. 19, pp. 35-43, 2015.

[27] T. Y. Kim, D. W. Seo, and S. H. Bae, "The study of bio emotion cognition follow stress index number by multiplex SVM algorithm," The Journal of Korea Institute of Information, Electronics, and Communication Technology, vol. 5, no. 1, pp. 45-51, 2012.

[28] S. Fazli, J. Mehnert, J. Steinbrink et al., "Enhanced performance by a hybrid NIRS-EEG brain computer interface," NeuroImage, vol. 59, no. 1, pp. 519-529, 2012.

[29] M. H. Lee, S. Fazli, J. Mehnert, and S. W. Lee, "Hybrid braincomputer interface based on EEG and NIRS modalities," in 2014 International Winter Workshop on Brain-Computer Interface (BCI), IEEE, Jeongsun-kun, South Korea, 2014.

[30] G. Peharz and A. Ulm, "Quantifying the influence of colors on the performance of c-Si photovoltaic devices," Renewable Energy, vol. 129, no. A, pp. 299-308, 2018.

[31] L. Y. Hu and H. Y. Fan, "Inversion formula and Parseval theorem for complex continuous wavelet transforms studied by entangled state representation," Chinese Physics B, vol. 19, no. 7, Article ID 074205, 2010.

[32] I. H. Bae, C. H. Kim, and H. T. Noh, "Design and evaluation of a fuzzy logic based multi-hop broadcast algorithm for IoT applications," KSII Transactions on Internet and Information Systems, vol. 17, no. 6, pp. 17-23, 2016.

[33] C. Wu, S. Ohzahata, Y. Ji, and T. Kato, "Joint fuzzy relays and network-coding-based forwarding for multihop broadcasting in VANETs," IEEE Transactions on Intelligent Transportation Systems, vol. 16, no. 3, pp. 1415-1427, 2014.

[34] K. Pat, “IEEE 802.15 WPAN ${ }^{\mathrm{TM}}$ Task Group 4," 2019, http:// ieee802.org/15/pub/TG4.html.

[35] T. W. Rauber, F. D. A. Boldt, and F. M. Varejãao, "Heterogeneous feature models and feature selection applied to bearing fault diagnosis," IEEE Transactions on Industrial Electronics, vol. 62, no. 1, pp. 637-646, 2015.

[36] S. U. Jan, Y. D. Lee, J. P. Shin, and I. S. Koo, "Sensor fault classification based on support vector machine and statistical time-domain features," IEEE Access, vol. 5, pp. 8682-8690, 2017.

[37] T. Y. Kim, B. H. Song, and S. H. Bae, "A design and implementation of music \& image retrieval recommendation system based on emotion," Journal of the Institute of Electronics Engineers of Korea, vol. 47, no. 1, pp. 73-79, 2010, UCI : G704A00451, 2010.47.1.002.

[38] H. J. Kim, S. H. Lee, and H. G. Lee, "Development of stress index model and u-SMC (stress management center) business model from the context-aware computing perspective," Journal of Intelligent Information System, vol. 14, no. 2, pp. 21-44, 2008, UCI : G704-000721, 2008.14.2.001.

[39] S. A. Hosseini, M. A. Khalilzadeh, and S. Changiz, "Emotional stress recognition system for affective computing based on biosignals," Journal of Biological Systems, vol. 18, no. 1, pp. 101-114, 2010.

[40] J. S. Kang, G. J. Jang, and M. H. Lee, "Stress status classification based on EEG signals," The Journal of the Institute of Internet Broadcasting and Communication, vol. 16, no. 3, pp. 103-108, 2016.

[41] L. Saeed, S. Shima, K. Reza, A. Alireza, and H. Boshra, "Support vector machine classification of brain states exposed to social stress test using EEG-based brain network measures," Biocybernetics and Biomedical Engineering, vol. 39, no. 1, pp. 199-213, 2019. 


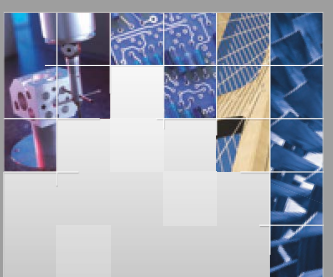

\section{Enfincering}
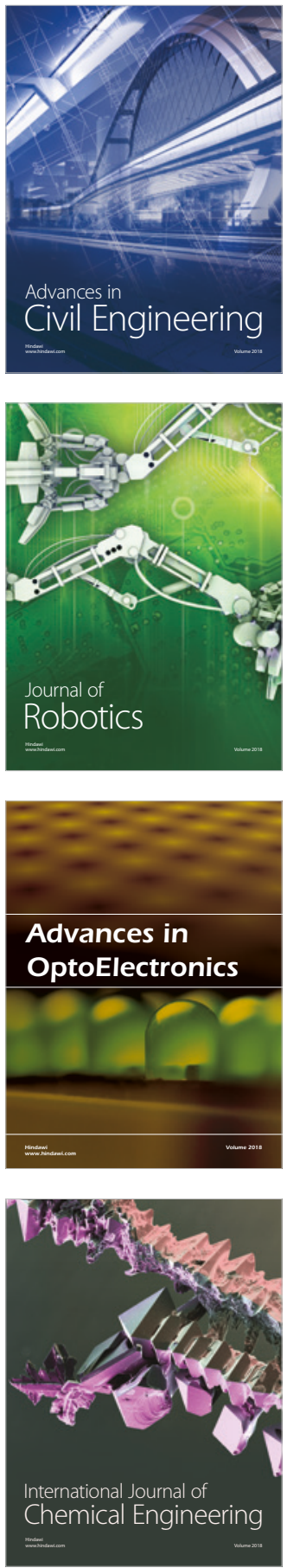

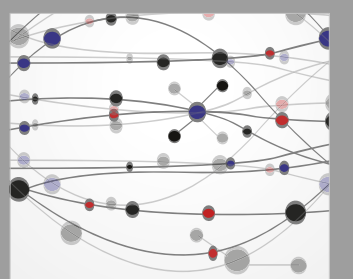

\section{Rotating \\ Machinery}

The Scientific World Journal

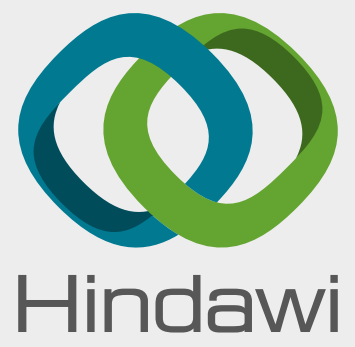

Submit your manuscripts at

www.hindawi.com
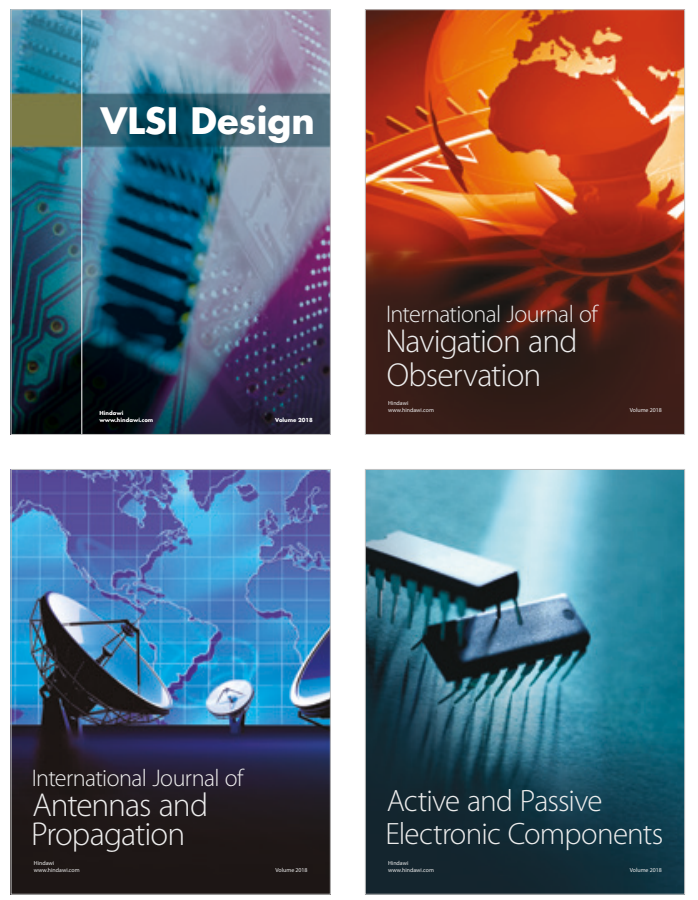
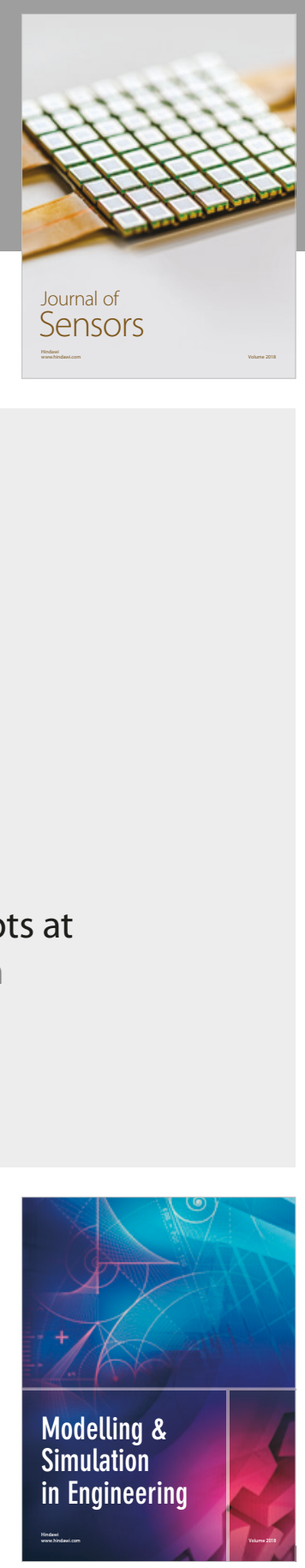

\section{Advances \\ Multimedia}
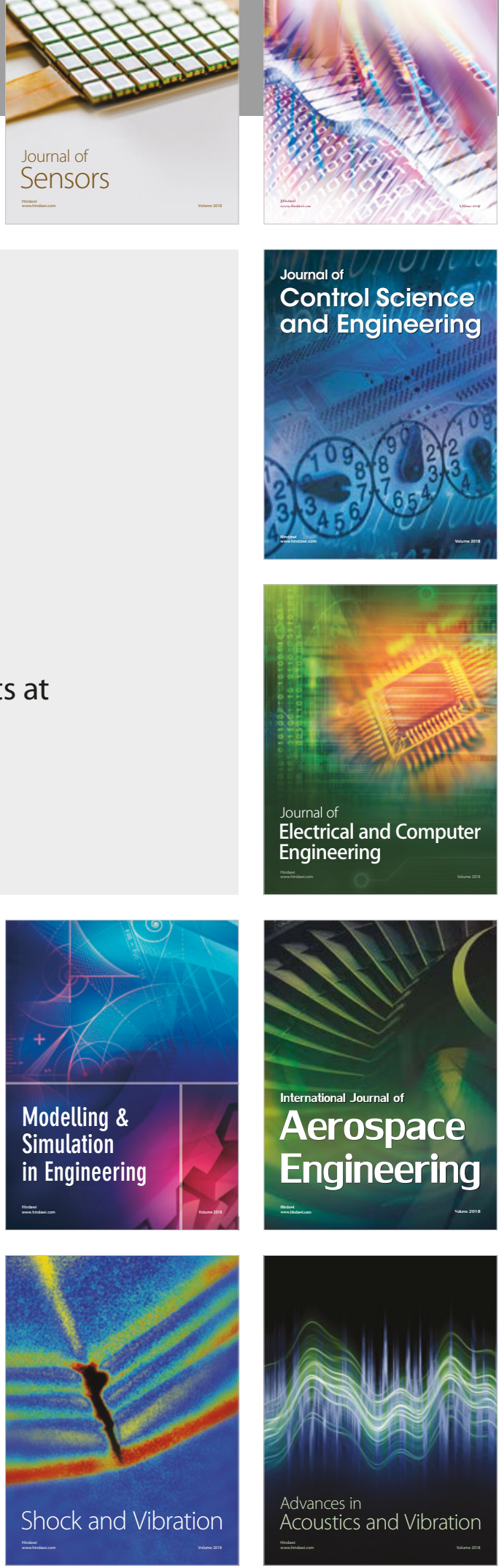